\title{
On the power of Public-key Function-Private Functional Encryption
}

\author{
Vincenzo Iovino ${ }^{1 *}$, Qiang Tang ${ }^{2}$, Karol Żebrowski ${ }^{3}$ \\ 1*University of Luxembourg, vinciovino@gmail.com \\ ${ }^{2}$ Luxembourg Institute of Science and Technology, qiang.tang@list.lu \\ ${ }^{3}$ University of Warsaw, k.zebrowski@mimuw.edu.pl
}

\begin{abstract}
:
In the public-key setting, known constructions of function-private functional encryption (FPFE) were limited to very restricted classes of functionalities like inner-product [Agrawal et al. - PKC 2015]. Moreover, its power has not been well investigated. In this paper, we construct FPFE for general functions and explore its powerful applications, both for general and specific functionalities.

One key observation entailed by our results is that Attribute-based Encryption with function privacy implies FE, a notable fact that sheds light on the importance of the function privacy property for FE.
\end{abstract}

\section{Introduction}

Functional Encryption (FE) [1] is a sophisticated type of encryption that allows fine-grained control over encrypted data. Progressively, more expressive forms of FE were constructed in a series of works (see, e.g., [2, 3, 4]) culminating in the breakthrough of Garg et al. [5] that put forth the first candidate FE for all poly-size Boolean circuits.

In a FE system, a central authority can hand a user with a token for any function $f$ in some functionality space; such token allows the user to compute $f(m)$ on a ciphertext encrypting $m$. The security notion in these works only takes in account the privacy of the message that dictates that beyond $f(m)$ no other information should be leaked beyond $m$. However, these works do not consider the security of the function. In other words, the token leaks $f$ in the clear. In the symmetrickey setting, a preliminary study of FE with function privacy was initiated by Shen et al. [6] for the inner-product functionality [4], subsequently followed by constructions for general functionalities [7]. Boneh et al. [8] put forward the study of public-key function-private FE providing constructions for the IBE functionality, then followed by works that considered the inner-product functionalities (and its variants) [9, 10].

Inevitably, the adversary can always try to infer partial information about the function in the token by using the public key to encrypt messages of his choice. For this reason, Boneh et al. [8] consider functions chosen from high min-entropy distributions. Precisely, in the context of IBE they propose an indistinguishability (IND) style real-or-random definition of function privacy, that stipulates that as long as the identity id was chosen from a sufficiently high min-entropy distribution, the adversary should not be able to discriminate the token for id from a token for a uniformly random identity. Agrawal et al. [10] strengthen simulation-based definitions for function privacy but assuming non-standard inefficient simulators; this is necessary due to broad impossibility results 
for simulation-secure FE (see Agrawal et al. for a survey).

It seems that a meaningful simulation-based security notion of public-key function-private functional encryption (FPFE) for some expressive enough class of Boolean circuits would imply virtual black box (VBB) obfuscation for the same class of circuits and thus it seems unachievable even for $\mathrm{NC}^{1}$ circuits. For such reasons, in this work we stick with the indistinguishability-based (INDbased) definition and defer to future works the study of stronger security notions.

Specifically, in the case of Boolean circuits, we consider what we call pairs of ensembles of efficiently samplable feasible entropy distributions, a strengthening of a notion defined by Agrawal et al. [10]. Roughly speaking, a pair of ensembles of distributions $D_{0}$ and $D_{1}$ over the same class of circuits are called a pair of ensembles of feasible entropy distributions if a circuit sampled from $D_{0}$ cannot be differentiated from a circuit sampled from $D_{1}$, given just oracle access to the circuit. Formal definition is given in Section 2.

Note that we put the constraint that the distributions be efficiently samplable. This is because, in the context of function privacy, as well as for functional anonymous signatures that we will introduce later, users sample the cryptographic objects from efficiently samplable distributions. This requirement makes possible assume what we call quasi-siO that weakens siO in that, whereas siO consider general (non necessarily efficiently samplable) distributions, quasi-siO only considers efficiently samplable ones. This subtle difference turns out to be very important; indeed it is the key to make such primitives composable.

To our knowledge no previous work in literature considered public-key FPFE for more general functionalities, like poly-sized circuits or even $\mathrm{NC}^{1}$ circuits. This leads to the main questions that we study in this work:

Can we achieve public-key FPFE for more general functionalities, like $\mathrm{NC}^{1}$ or even all poly-sized circuits, from reasonable assumptions? And what applications and other primitives can we build from FPFE (not necessarily for general functionalities)?

Based on the existence of quasi-siO proposed by Bitansky, Canetti, Kalai and Paneth [11], ${ }^{1}$ we answer affirmatively to the first question. The solution we propose is conceptually simple and elegant but we believe that the key is in having discovered and identified quasi-siO as the main building block, a relation that was not known before in the literature.

Note that quasi-siO is a weakened version of strong $\mathrm{iO}(\mathrm{siO})$, which guarantees that no efficient adversary can distinguish two feasible entropy distributions $D_{0}$ or $D_{1}$. The weakening lies in the fact that quasi-siO requires the distributions to be efficiently samplable.

We answer the second question by mainly demonstrating the implication with respect to functional anonymous signatures, FE for randomized functionalities, and adaptive security for efficient Boolean formulae encryption; for this application we do not require FPFE for general functionalities). Some of our results are not technically involved, but this is a due to our recognition of the power of these primitives not studied so far, and some applications we derive from them improve the state of the art in the field or solve known problems. Thus, we deem the simplicity of our approach a positive feature not a shortcoming.

Our results are not only an example of the power and of the applications of FPFE but also and mainly of the power siO/quasi-siO, and in Section 6 we show equivalences between them. We mention that recently the existence of $\mathrm{siO}$ was put in contention with the existence of other strong assumptions in [12] and we defer the reader to the discussion after Definition 2.2 for further details. Anyhow, we point out that all our results based on quasi-siO can be instantiated for $\mathrm{NC}^{1}$ circuits

\footnotetext{
${ }^{1}$ The name quasi-siO is ours. The authors define a weakening of the their notion of siO (see the following) without explicitly naming it.
} 
assuming only quasi-siO for $\mathrm{NC}^{1}$ circuits.

\subsection{Public-key FPFE based on Quasi-siO}

It is worthy reminding why existing constructions of FE do not offer any meaningful function privacy. Consider the construction of Garg et al. [5] of FE from iO. Therein, the token for a circuit $C$ consists of an $\mathrm{iO}$ of $C$. One could hope that being the circuit obfuscated it should hide as much information as possible about the circuit. Nonetheless, we argue that the form of function privacy attained here is very limited. Indeed, the token for $C$ is indistinguishable from the token for any other functionally equivalent circuit $C^{\prime}$ but may leak any other sensitive information.

We now show that this is insufficient in concrete applications. Consider the case of circuits implementing point functions. Specifically, for any binary string $x \in\{0,1\}^{n}$ consider the class of circuits $\mathcal{C}_{x}$ that contain all circuits $C$ defined so that $C$ on input a binary string $y$ of length $n$ outputs 1 if and only if $y=x$. Then, the class of circuits implementing point functions, let us say restricted to points of length $n$, is the union of all $\mathcal{C}_{x}$ 's for all strings $x$ of length $n$. It is trivial to notice that an $\mathrm{iO}$ for this class could just return the value $x$ in clear, ${ }^{2}$ assuming that this can be done efficiently. That is, the (non necessarily efficient) obfuscator that on input a circuit $C \in \mathcal{C}_{x}$ for some $x \in\{0,1\}^{n}$ outputs $x$ in the clear (with evaluation procedure associated in the obvious way) is provably an $\mathrm{iO}$. We claim that this obfuscator, when used to construct FE, does not offer any guarantee of function privacy for these classes of functions.

In fact, consider two distributions $D_{0}$ and $D_{1}$ over strings in $\{0,1\}^{n}$ defined so that the first bit in the strings drawn from $D_{b}$, for $b \in\{0,1\}$ is $b$ and the remaining bits are uniformly and independently chosen. Then, a token for a point $x$ drawn from $D_{0}$ can be easily distinguished from a token for a point drawn from $D_{1}$. This is because the obfuscated point leaks $x$ in clear and looking just at the first bit of it, the token can be distinguished. The above analysis motivate us to use siO. If the token was instead a siO of the circuit, it would leak as little information as possible about the circuit. To the aim of having conceptually simple and general constructions, we construct a FPFE scheme by nesting a generic FE scheme (without function privacy) with a siO.

Specifically our FPFE scheme FPFE will use the underlying FE scheme FE as a black-box and will have identical procedures except that a token for a circuit $C$ will consist of a token of FE for the circuit qsiO $(C)$, where qsiO is a quasi-siO. That is, setting $C^{\prime}=$ qsiO $(C)$, a token of FPFE for $C$ will be a token of FE for $C^{\prime}$.

The intuition is that, even though this token is computed with a non function-private scheme, it is built on the top of a circuit obfuscated with quasi-siO, and thus it should leak as little information as possible about the function. In fact, we confirm this intuition providing reductions to quasi-siO and FE.

Note here that the underlying FE scheme guarantees the privacy of the encrypted messages and quasi-siO is only used to add the extra layer of function privacy.

The modularity of our approach allows to instantiate a FPFE for a class of circuits $C$ assuming only a quasi-siO for the same class of circuits assuming that the class $\mathcal{C}$ is enough expressive, specifically includes at least all $\mathrm{NC}^{1}$ circuits. Furthermore, the construction generalizes easily to multi-inputs FE (MIFE, in short) [13] allowing to construct the first MIFE scheme with function privacy (FPMIFE, in short). The definition of a FPFE scheme and its security are presented in Section 2.3 and its construction from quasi-siO is presented in Section 3.

The reverse direction also holds. In fact, a quasi-siO qsiO for class of circuits $\mathcal{C}$ can be constructed

\footnotetext{
${ }^{2}$ Precisely, we also have to define a corresponding evaluation procedure in the obvious way.
} 
from a FPFE scheme FPFE for the same class in the following way. For any input $C$ the algorithm qsiO $(C)$ outputs the public-key of the FPFE scheme and a token Tok for $C$ of FPFE. To evaluate such obfuscated circuit on an input $x$, the evaluation algorithm associated with qsi $\mathcal{O}$ takes as input the public-key and Tok and encrypts $x$ to get $\mathrm{Ct}$ and evaluates Tok on $\mathrm{Ct}$ to get $C(m)$. The correctness of FPFE and its INDFP-Security defined in Section 2.3 imply that such obfuscator is a quasi-siO. This construction also reaffirms that a meaningful simulation-based security notion for FPFE for a class $\mathcal{C}$ would imply VBB obfuscation for $\mathcal{C}$, and thus is unachievable in general. For such reason we stick with an IND-based definition of function privacy.

\subsection{Functional Anonymous Signatures}

As warmup we construct from FPFE a new primitive called Functional Anonymous Signature (FAS). Recall that the Naor's transformation (presented in [14]) allows to transform an identitybased encryption (IBE) scheme. The transformation is based on the idea that the token for an identity id acts as a signature for it. Such signature can then be verified by encrypting the pair $(r$, id) for a random string $r$ and testing whether the token (i.e., the signature) evaluated on such ciphertext returns $r$. By the security property of IBE, one can prove the unforgeability of signatures. We generalize this concept to FE and propose what we call FAS. With FAS, a user Alice can sign a Boolean circuit $C$ allowing Bob holding an input $m$ to verify (1) that the signature was issued by Alice and that (2) $C(m)=1$.

This makes no sense since in general the signature leaks the signed input, thus Bob could verify that $C(m)=1$ by himself without running the verification procedure at all. We envision a scenario where the signature of Alice of a circuit $C$ hides $C$ if it is drawn from a feasible entropy distribution. In this case, the intent of Bob is to verify (1) that Alice signed some circuit $C$, that is not known to him, and (2) verify that his input $m$ satisfies the circuit, e.g., $C(m)=1$.

We foresee FAS to be a very useful primitive in practice, e.g. in the following authenticated policy verification mechanism. Alice, the head of a company, can publish her verification key and with the corresponding secret key can sign an hidden policy $P$ chosen from some known distribution $D$ and send the signature $\sigma$ of $P$ to the server of her company. The secretary of the company, who is assumed to be honest but curious, can grant Bob access to some private document iff the access pattern $m$ held by Bob verifies the signature of Alice, and in particular her hidden policy, i.e., $P(m)=1$. If the signature is verified by the access pattern of Bob, then the secretary has the guarantee that (1) the policy was signed by Alice and (2) the access pattern of Bob satisfies such policy.

Both Bob and the secretary have no information about the policy except what can be trivially inferred from the distribution $D$. Due to the possibility of using universal circuits in FAS, the role of access pattern and policy can be inverted, that is Alice can sign an access pattern and Bob holding a policy can verify whether his policy satisfies her access pattern. It is easy to see that FAS implies traditional signature schemes.

We define FAS with a notion that we call functional unforgeability, that suits for most applications of FAS. The notion does not consider as valid the forgery of a circuit more restricted than a circuit for which a signature was seen. ${ }^{3}$

To see why such condition is not too restrictive, consider the above application. In that case, the security of FAS should prevent some unauthorized user to claim that Alice signed a document who authorizes him. This is exactly what the condition states. Note also that being Alice semi-trusted

\footnotetext{
${ }^{3}$ That is, it is not considered as a valid forgery if an adversary given a signature of circuit $C$ can sign another circuit $C^{\prime}$ that computes the same function as $C$ or is more restricted than $C$.
} 
we do not consider a breach of security if she is able to forge a signature for a circuit $C^{\prime}$ more restricted than the circuit $C$ of which she received a signature from Alice (a circuit $C^{\prime}$ is said to be more restricted than $C$ if $C^{\prime}(x)=1$ implies $C(x)=1$ ). Only malicious users have the interest to forge new signatures and in this case their scope is to forge signatures for circuits that authorize them, so a forgery for a more restricted circuit (or a functionally equivalent one) must not be considered a successful attack.

For some applications such security could not suffice but we show that it is possible to make FAS unforgeable according to the classical notion of unforgeability (i.e., requiring that any PPT adversary can not forge a signature for a circuit $C^{\prime}$ different (as bit string) from any circuit $C$ for which it saw a signature) just adding a traditional unforgeable scheme on the top of it. Beyond unforgeability, we require anonymity, namely that a signature $\sigma$ hide as much information as possible about $C$ except what can be inferred from knowledge of the distribution from which $C$ is drawn.

FPFE fits perfectly in the picture, and in fact we show that it implies FAS in a black-box way. Specifically, we extend the Naor's transformation to construct FAS for a class of circuits $\mathcal{C}$ from Attribute-based Encryption (ABE) [15] with function privacy, a weaker notion of FPFE, for the same class $\mathcal{C}$.

Despite of the name, FAS is not related at all with functional signatures of Goldwasser et al. [16]. Primitives more related to FAS are content-concealing signatures and confidential signatures $([17,18])$ that can be viewed as a weak form of FAS schemes without functional capabilities (or alternatively for the class of equality predicates). The definition of FAS, its security and its construction from ABE with function privacy (FPABE) are presented in Section 4.

We mention that it is possible to construct FAS in a more direct way from quasi-siO, but our aim is also to show equivalences among FAS, quasi-siO and FPFE (see Section 6).

\subsection{Functional Encryption for Randomized Functionalities}

Goyal et al. [19] introduce the concept of FE for randomized circuits. In this setting, the challenge is to guarantee that the circuit be evaluated on fresh randomness that can not be maliciously chosen. A first attempt to the problem would be to include the seed of a pseudo-random function in the token. Unfortunately, this approach fails since the token is not guaranteed to hide the function that the circuit is supposed to compute.

This leaves open the possibility that this basic idea could work assuming a FE whose token hides the function (i.e., with function privacy), and in fact we are able to confirm this intuition by showing a black-box construction of FE for randomized circuits (RFE) from FPFE for (deterministic) circuits. We adopt an indistinguishability-based security for RFE, but unlike Goyal et al. we do not take in account the problem of dishonest encryptors that goes beyond the scope of our work. (We note that the problem of dishonest encryptions is a concern not only for RFE but for FE and FPE as well.)

Our construction of RFE also preserves the function privacy of the underlying FPFE and thus satisfies the standard notion of function privacy where the adversary can ask distributions of deterministic circuits. We call this notion FPRFE. We believe that it also satisfy a form of function privacy extended in a natural way to support randomized circuits, but we did not investigate the details.

Our construction of RFE can be easily extended to the multi-inputs setting, resulting in the first construction, assuming only quasi-siO, of a FPMIFE for randomized functionalities (as said before, where the function privacy is restricted to deterministic circuits) with selective form of security. The restriction of selective security can be removed assuming in addition an adaptively-secure 
MIFE.

The definition of RFE and its security are presented in Section 2.4 and its construction from FPFE is presented in Section 5.

\subsection{Adaptively-secure FE for CNF/DNF formulae of bounded degree}

Henceforth, we assume familiarity with inner-product encryption (IPE) introduced by Katz et al. [4].

Katz et al. show how to construct from IPE polynomial evaluation from IPE and FE for a subclass of Boolean formulae with a bounded number of variables (BoolEnc). Hereafter, we focus on DNF formulae encryption (DNFEnc). Analogous considerations hold for other classes of Boolean formulae that can be derived from IPE, e.g., CNF formulae.

Conjunctions can be handled in the following way. Consider the predicate $\operatorname{AND}_{I_{1}, I_{2}}$ where $\operatorname{AND}_{I_{1}, I_{2}}\left(x_{1}, x_{2}\right) \triangleq 1$ if both $x_{1}=I_{1}$ and $x_{2}=I_{2}$. Then, we can choose a random $r \leftarrow \mathbb{Z}_{p}$ (here we assume that the coefficient of the polynomial are over $\mathbb{Z}_{p}$ ) and letting the token correspond to the polynomial $p\left(x_{1}, x_{2}\right) \triangleq r \cdot\left(x_{1}-I_{1}\right)+\left(x_{2}-I_{2}\right)$. If $\operatorname{AND}_{I_{1}, I_{2}}\left(x_{1}, x_{2}\right)=1$ then $p\left(x_{1}, x_{2}\right)=$ 0 , whereas if $\operatorname{AND}_{I_{1}, I_{2}}\left(x_{1}, x_{2}\right)=0$ then, with all but negligible probability over the choices of $r$, it will hold that $p\left(x_{1}, x_{2}\right) \neq 0$. Disjunctions can be implemented by defining a polynomial

$p^{\prime}\left(x_{1}, x_{2}\right) \triangleq\left(x_{1}-I_{1}\right) \cdot\left(x_{2}-I_{2}\right)$. Conjunctions and disjunctions can be combined to get general DNF formulae but, as the Katz et al.'s transform has a super-polynomially growth, we have to put a bound on the number of variables.

As observed by Katz et al. in general the token may leak the value of $r$ in which case the adversary will be able to find $x_{1}, x_{2}$ such that $\operatorname{AND}_{I_{1}, I_{2}}\left(x_{1}, x_{2}\right)=0$ yet $p\left(x_{1}, x_{2}\right)=0$. Since, however, they consider the "selective" notion of security, this is not a problem in their setting. On the other hand, disjunctions can be handled without issues.

Anyhow, an adaptively-secure IPE schemes [20] can not be directly employed in this transformation and thus to construct an adaptively-secure DNFEnc. FPFE turns out to be useful in this context: assuming that the underlying IPE satisfies our notion of function privacy, we show that an adaptively-secure IPE with function privacy implies an adaptively-secure DNFEnc. The idea is that a function-private scheme hides the value $r$ so that the adversary cannot make the reduction to fail. In Appendix B.1 we provide a rigorous proof of this fact.

It is out of the scope of this work to provide concrete instantiations of function-private schemes for our transformation but our result emphasizes the importance of function-privacy even for practical matters. For instance, the IPE scheme of Agrawal et al. [21] is clearly subject to functionprivacy attacks and thus cannot be employed in the Katz et al.'s transformation whereas, though not backed by any security proof, the IPE schemes of Katz et al. does not seem subject to any of such attacks. Our result suggests that care has to be taken when instantiating the Katz et al.ś transformation.

\subsection{Relation between primitives}

In Section 6, we present a general picture of the relations among all these related primitives. One key observation is that Attribute-based Encryption with function privacy implies FE, a notable fact that sheds light on the importance of the function privacy property for FE. 


\section{Definitions}

\subsection{Preliminaries}

In our work, we make use of the following definition inspired by a similar definition from Agrawal et al. $[22,10]$.

Definition 2.1. [Pair of Ensembles of Feasible Entropy Distributions]. Let $D_{0}=\left\{D_{0, n}\right\}_{n \in \mathbb{N}}$ and $D_{1}=\left\{D_{1, n}\right\}_{n \in \mathbb{N}}$ be two ensembles of distributions over a class of circuits $\mathcal{C}=\left\{\mathcal{C}_{n}\right\}_{n \in \mathbb{N}}$ where any $n \in \mathbb{N}, \mathcal{C}_{n}$ contains circuits of same size. We say that $D_{0}$ and $D_{1}$ are a pair of ensembles of feasible entropy distributions, if for all non-uniform families of (possibly inefficient) algorithms $\mathcal{A}=\left\{\mathcal{A}_{n}\right\}_{n \in \mathbb{N}}$ making a polynomial number of queries to its oracle, it holds that:

$$
\left|\operatorname{Pr}_{C \leftarrow D_{0}}\left[\mathcal{A}_{n}^{C(\cdot)}\left(1^{n}, 1^{|C|}\right)=1\right]-\operatorname{Pr}_{C \leftarrow D_{1}}\left[\mathcal{A}_{n}^{C(\cdot)}\left(1^{n}, 1^{|C|}\right)=1\right]\right| \leq \operatorname{negl}(n) .
$$

Note that in the above definition we do not require that the distributions be efficiently samplable but for all our applications we will put such additional constraint. So we will talk about a pair of ensembles of efficiently samplable feasible entropy distributions with the obvious meaning. In this work, we make use of puncturable pseudorandom functions [23] which are essentially pseudorandom functions (PRFs, in short) that can be defined on all inputs except for a polynomial number of inputs. We refer the reader to [23] the formal definitions.

We refer the reader to Appendix A for the standard definitions of FE and its IND-Security.

\subsection{Strong and Quasi-strong Indistinguishability Obfuscation}

Strong indistinguishability obfuscation has been introduced by Bitansky et al. [11]. Their formulation is syntactically different from ours, but as they point out ([24], p. 4) it is equivalent to ours. Thus, without loss of generality we adopt the following formulation as it is more suitable for our scopes.

Definition 2.2. [Strong Indistinguishability Obfuscators for Circuits] A uniform PPT machine siO is called a strong indistinguishability obfuscator ( $\mathrm{siO}$, in short) for a circuit family $\mathcal{C}=\left\{\mathcal{C}_{n}\right\}_{n \in \mathbb{N}}$, if the following conditions are satisfied:

- Correctness: $\forall n, \forall C \in \mathcal{C}_{n}, \forall x \in\{0,1\}^{\star}$ we have

$$
\operatorname{Pr}\left[C^{\prime}(x)=C(x): C^{\prime} \leftarrow \operatorname{siO}\left(1^{n}, C\right)\right]=1 .
$$

- Strong indistinguishability: For all pairs of ensembles of feasible entropy distributions $D_{0}=$ $\left\{D_{0, n}\right\}_{n \in \mathbb{N}}$ and $D_{1}=\left\{D_{1, n}\right\}_{n \in \mathbb{N}}$ over a class of Boolean circuits $\mathcal{C}^{\prime}=\left\{\mathcal{C}_{n}^{\prime}\right\}_{n \in \mathbb{N}} \subset \mathcal{C}$ where for any $n \in \mathbb{N}$ the set $\mathcal{C}_{n}^{\prime}$ contains circuits of the same size, for any non-uniform family of PPT distinguishers $\mathcal{D}=\left\{\mathcal{D}_{n}\right\}_{n \in \mathbb{N}}$, there exists a negligible function negl $(\cdot)$ such that the following holds: For all $n \in \mathbb{N}$, we have that

$\left|\operatorname{Pr}_{C \leftarrow D_{0, n}}\left[\mathcal{D}_{n}\left(1^{n}, 1^{|C|}, \operatorname{siO}\left(1^{n}, C\right)\right)=1\right]-\operatorname{Pr}_{C \leftarrow D_{1, n}}\left[\mathcal{D}_{n}\left(1^{n}, 1^{|C|}, \operatorname{siO}\left(1^{n}, C\right)\right)=1\right]\right| \leq \operatorname{neg}(n)$.

Bitansky et al. also hint the following weakening of siO (as they do not explicitly assign a name to the primitive, the new name is ours).

Definition 2.3. [Quasi-strong indistinguishability Obfuscators for Circuits] A quasi-strong indistinguishability obfuscator (quasi-siO, in short) for a circuit family $\mathcal{C}$ is defined analogously to siO except that the strong indistinguishability condition is weakened with the quasi-strong indistinguishability condition that is identical to the former except that it is required that the ensembles of distributions be ensembles of efficiently samplable distributions. 


\subsection{Function-Private Functional Encryption (FPFE)}

A FPFE scheme is a FE scheme satisfying IND-Security and the following additional security notion. Indistinguishability-based function privacy security. The IND-based function privacy notion of security for a functional encryption scheme FPFE = (Setup, KGen, Enc, Eval) for a class of circuits $\mathcal{C}=\left\{\mathcal{C}_{\lambda}\right\}_{\lambda}$ is formalized by means of the following game INDFP ${ }_{\mathcal{A}}^{\mathrm{FPFE}}$ between an adversary $\mathcal{A}=\left(\mathcal{A}_{0}, \mathcal{A}_{1}\right)$ and a challenger $\mathcal{C}$. Below, we present the definition for only one function; it is easy to see the definition extends naturally for multiple functions.

$\operatorname{INDFP}_{\mathcal{A}}^{\mathrm{FPFE}}\left(1^{\lambda}\right)$

1. $\mathcal{C}$ generates $(M p k, M s k) \leftarrow \operatorname{Setup}\left(1^{\lambda}\right)$ and runs $\mathcal{A}_{0}$ on input Mpk;

2. $\mathcal{A}_{0}$ submits queries for Boolean circuits $C_{i} \in \mathcal{C}_{\lambda}$ for $i=1, \ldots, q_{1}$ and, for each such query, $\mathcal{C}$ computes Tok $_{i}=\mathrm{KGen}\left(\mathrm{Msk}, C_{i}\right)$ and sends it to $\mathcal{A}_{0}$.

When $\mathcal{A}_{0}$ stops, it outputs two challenge distributions $D_{0, \lambda}, D_{1, \lambda}$ over $\mathcal{C}_{\lambda}$ and its internal state st.

3. $\mathcal{C}$ picks $b \in\{0,1\}$ at random, picks a circuit $C$ according to distribution $D_{b, \lambda}$, and computes the challenge token Tok $=\mathrm{KGen}(\mathrm{Msk}, C)$ and sends Tok to $\mathcal{A}_{1}$ that resumes its computation from state st.

4. $\mathcal{A}_{1}$ submits queries for circuits $C_{i} \in \mathcal{C}_{\lambda}$ for $i=q_{1}+1, \ldots, q$ and, for each such query, $\mathcal{C}$ computes $\operatorname{Tok}_{i}=\mathrm{KGen}\left(\mathrm{Msk}, C_{i}\right)$ and sends it to $\mathcal{A}_{1}$.

5. When $\mathcal{A}_{1}$ stops, it outputs $b^{\prime}$.

6. Output: if $b=b^{\prime}$ then output 1 else output 0 .

The advantage of adversary $\mathcal{A}$ in the above game is defined as

$$
\operatorname{Adv}_{\mathcal{A}}^{\mathrm{FPFE}, \mathrm{INDFP}}\left(1^{\lambda}\right)=\left|\operatorname{Prob}\left[\operatorname{INDFP}{ }_{\mathcal{A}}^{\mathrm{FPFE}}\left(1^{\lambda}\right)=1\right]-1 / 2\right| .
$$

Note that we did not put any non-trivial constraint on the above game. In fact, any PPT could trivially win in it. As in Agrawal et al. we need to restrict the class of adversaries to what are called legitimate function privacy adversaries.

Definition 2.4. A non-uniform family of PPT algorithms $\mathcal{A}=\left\{\mathcal{A}_{\lambda}\right\}_{\lambda \in \mathbb{N}}$ is called a legitimate function privacy adversary against a FPFE scheme for a class of circuits $\mathcal{C}=\left\{\mathcal{C}_{\lambda}\right\}_{\lambda \in \mathbb{N}}$ if all pairs of distributions $D_{0, \lambda}$ and $D_{1, \lambda}$ output by $\mathcal{A}_{\lambda}$ in the above game for security parameter $\lambda$ are such that $D_{0} \triangleq\left\{D_{0, \lambda}\right\}_{\lambda \in \mathbb{N}}$ and $D_{1} \triangleq\left\{D_{1, \lambda}\right\}_{\lambda \in \mathbb{N}}$ are of a pair of ensembles of efficiently samplable feasible entropy distributions ${ }^{4}$ over a circuit class $\mathcal{C}^{\prime}=\left\{\mathcal{C}_{\lambda}^{\prime}\right\}_{\lambda \in \mathbb{N}}$ where for any $\lambda \in \mathbb{N}, \mathcal{C}_{\lambda}^{\prime}$ contains circuits of the same size.

Definition 2.5. We say that FPFE is indistinguishability function private secure (INDFP-Secure, for short) if every legitimate function privacy adversary $\mathcal{A}=\left\{\mathcal{A}_{\lambda}\right\}_{\lambda \in \mathbb{N}}$ have at most negligible advantage in the above game. ${ }^{5}$

\footnotetext{
${ }^{4}$ Note that the adversary is randomized so that the distributions could depend on its randomness. Thus, the interpretation here is that all pairs of sequences $\left(D_{0, \lambda}, D_{1, \lambda}\right)_{\lambda \in \mathbb{N}}$, formed putting for any $\lambda$ some pair of distributions $D_{0, \lambda}$ and $D_{1, \lambda}$ that it is a possible (i.e., such that the adversary outputs them with non-zero probability) output of the adversary in the experiment for parameter $\lambda$, is a pair of ensembles of efficiently samplable feasible entropy distributions. Note that Agrawal et al. do not explicitly expand on this detail. Same considerations hold for later definition of FAS legitimate adversaries.

${ }^{5}$ Hereafter, we say that a family of algorithms $\mathcal{B}=\left\{\mathcal{B}_{n}\right\}_{n \in \mathbb{N}}$ has negligible advantage in a experiment if there exists a negligible function negl(.) such that for all $n \in \mathbb{N}$ the advantage of $\mathcal{B}_{n}$ in the experiment is at most negl $(n)$.
} 


\subsection{Functional Encryption for Randomized Functionalities}

Goyal et al. [19] introduced the concept of FE for randomized functionalities. Like in Komargodski et al. [25] in this paper we do not take in account the problem of dishonest decryptors; this problem does not arise only in the context of randomized functionalities, and we think it go beyond the scope of our paper. A FE for randomized functionalities (RFE, in short) has the same syntax of a FE scheme for deterministic functionalities, with the obvious change that the functionality takes two inputs, the message and the randomness. We refer to the aforementioned papers for details. In this paper we will focus on the functionality of randomized circuits, both randomized $\mathrm{NC}^{1}$ circuits and general randomized poly-size circuits, defined in an anologous way to the deterministic case except that such circuits also take a random string as second input. We refer the reader to Goyal et $a l$. for formal definitions of RFE. As our formalization of security for RFE we choose what Goyal et al. call "security against key queries after public-key" except that, as discussed before, we do not take in account dishonest decryptors.

\section{Construction of FPFE from quasi-siO}

Definition 3.1. [quasi-siO-Based Construction] Let qsiO be a quasi-siO and $\mathrm{FE}=$ (FE.Setup, FE.Enc, FE.KGen, FE.Eval) be a FE scheme, both for an enough expressive class of circuits $\mathcal{C}$ (at least all $\mathrm{NC}^{1}$ circuits). We define a $\mathrm{FPFE}$ scheme FPFE[qsiO, FE] = (Setup, KGen, Enc, Eval) for the class of circuits $\mathcal{C}$.

- Setup $\left(1^{\lambda}\right)$ : output the public-key Mpk and master secret-key Msk computed, respectively, as the public-key and the master secret-key output by FE.Setup $\left(1^{\lambda}\right)$.

- $\operatorname{Enc}(\mathrm{Mpk}, m)$ : output $\mathrm{Ct} \leftarrow$ FE.Enc $(\mathrm{Mpk}, m)$.

- KGen(Msk, $C$ : output the token FE.KGen(Msk, qsiO $(C))$.

- Eval(Mpk, Ct, Tok): output FE.Eval(Mpk, Ct, Tok).

It is easy to see that the scheme satisfies correctness assuming the correctness of qsiO and FE, and the following theorem holds.

Theorem 3.2. If FE is IND-Secure then FPFE $[q s i \mathcal{O}, F E]$ is IND-Secure.

The proof of the next theorem is given in Appendix B.2.

Theorem 3.3. If qsiO is a quasi-siO then FPFE $[q s i \mathcal{O}, \mathrm{FE}]$ is INDFP-Secure.

\section{Construction of FAS from FPABE}

Overview. The construction extends the Naor's transformation from IBE to (traditional) signature schemes. Specifically a token for a circuit $C$ computed with the ABE system acts as a signature for $C$. The security of the ABE system guarantees the unforgeability of FAS: no adversary, given a token for circuit $C$ can produce another token for another circuit that would enable to distinguish the encryption of two ciphertexts computed with an attribute $x$ such that $C(x)=0$. If in addition the ABE system is function private, the resulting FAS scheme is anonymous as well.

Definition 4.1. [FPFE-Based Construction] Let FPABE = (FPABE.Setup, FPABE.Enc, FPABE.KGen, FPABE.Eval) be a FPABE scheme for the class of Boolean circuits 
$\mathcal{C}=\left\{\mathcal{C}_{n}\right\}_{n \in \mathbb{N}}$. We define a FAS scheme FAS[FPABE $]=($ FAS.Setup, FAS.Sign, FAS.Verify) for $\mathcal{C}$ as follows.

- FAS.Setup $\left(1^{\lambda}\right)$ : set verification key vk and signing key sk to be respectively the public-key and the master secret-key output by the setup of FPABE .

- FAS.Sign (sk, C): output $\sigma \leftarrow$ FPABE.KGen(sk, C).

- FAS.Verify $(\mathrm{vk}, \sigma, \mathrm{x})$ : choose random value $r \leftarrow\{0,1\}^{\lambda}$, encrypt $\mathrm{Ct} \leftarrow \mathrm{FPABE}$.Enc $(\mathrm{vk},(r, x))$ and compute $r^{\prime} \leftarrow$ FPABE.Eval $(\mathrm{vk}, \mathrm{Ct}, \sigma)$. If $r^{\prime}=r$ then output 1 otherwise output $\perp$.

It is easy to see that the scheme satisfies correctness assuming the correctness of FPABE and is functionally unforgeable. ${ }^{6}$ In fact an adversary outputting a forgery that satisfies the winning condition of functional unforgeability, is a valid adversary against the security of FPABE and thus as in the Naor's transformation the forgery can be used to break the security of FPABE. Thus, the following theorem holds.

Theorem 4.2. If FPABE is IND-Secure then FAS[FPABE] is unforgeable.

The proof of the following theorem is given in Appendix B.3.

Theorem 4.3. If FPABE is INDFP-Secure then FAS[FPABE] is anonymous.

\section{Construction of RFE from FPFE}

Definition 5.1. [FPFE-Based Construction] Let $F=(F . K e y, F . P n c t, F . E v a l)$ be a puncturable pseudorandom function and

FPFE $=$ (FPFE.Setup, FPFE.Enc, FPFE.KGen, FPFE.Eval) be a FPFE scheme, both for a sufficiently expressive class of (deterministic) Boolean circuits $\mathcal{C}^{\prime}$. We define a RFE scheme RFE $[F, F P F E]=$ (Setup, KGen, Enc, Eval) for the class of randomized Boolean circuits $\mathcal{C}=\left\{\mathcal{C}_{n}\right\}_{n \in \mathbb{N}}$ induced by $\mathcal{C}^{\prime}$ as follows.

- Setup $\left(1^{\lambda}\right)$ : generate Mpk and Msk computed, respectively, as the public-key and the master secretkey output by FPFE.Setup $\left(1^{\lambda}\right)$.

- Enc $($ Mpk, $m)$ : output Ct $\leftarrow$ FPFE.Enc $(M p k, m)$.

- KGen(Msk, $C$ ): on input Msk, a randomized circuit $C \in \mathcal{C}_{\lambda}$ with input of length $n$ and randomness of length $n$, compute $k \leftarrow \mathrm{F}$.Key $\left(1^{\lambda}\right)$ and output the token FPFE.KGen(Msk, $\left.C[k]\right)$ ) for the following deterministic circuit $C[k] \in \mathcal{C}_{2 \lambda}^{\prime}$.

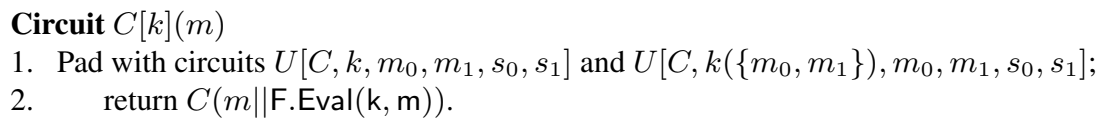

- Eval(Mpk, Ct, Tok): output FPFE.Eval(Mpk, Ct, Tok).

Correctness. It is easy to see that the scheme satisfies correctness assuming the correctness of FPFE and the pseudorandomness of $F$.

\section{Security reduction.}

\footnotetext{
${ }^{6}$ It is easy to make the above scheme even secure according to the traditional notion of unforgeability. It is sufficient to use a traditional unforgeable signature scheme and signing the token with such scheme. The resulting scheme will be unforgeable (according to the traditional notion) as well.
} 
Theorem 5.2. If FPFE is IND-Secure and INDFP-Secure, and F is a puncturable pseudorandom function, then RFE[F, FPFE] is INDRFE-Secure.

Proof: We reduce the security of our RFE scheme to that of the underlying primitives (FPFE and puncturable pseudorandom functions) via a series of hybrid experiments against a PPT legitimate RFE adversary $\mathcal{A}$ attacking the INDRFE-Security of RFE[F, FPFE].

- $H_{0}$. This corresponds to the INDRFE-Security game in which the challenge ciphertext encrypts the message $m_{0}$.

- $H_{1}$. This experiment is identical to $H_{0}$ except that any token for randomized circuit $C$ is computed as FPFE.KGen(Msk, $\left.U\left[C, k, m_{0}, m_{1}, s_{0}, s_{1}\right]\right)$ where $s_{b}=$ F.Eval $\left(\mathrm{k}, \mathrm{m}_{\mathrm{b}}\right)$ for $b \in$ $\{0,1\}$ and $U\left[C, k, m_{0}, m_{1}, s_{0}, s_{1}\right]$ is the following deterministic circuit:

Circuit $U\left[C, k, m_{0}, m_{1}, s_{0}, s_{1}\right](m)$

1. Pad with circuits $C[k]$ and $U\left[C, k\left(\left\{m_{0}, m_{1}\right\}\right), m_{0}, m_{1}, s_{0}, s_{1}\right]$;

2. if $m=m_{0}$ return $C\left(m \| s_{0}\right)$;

2. else if $m=m_{1}$ return $C\left(m \| s_{1}\right)$;

3. otherwise return $C(m \| \mathrm{F}$.Eval $(\mathrm{k}, \mathrm{m}))$.

Claim 5.3. Indistinguishability of $H_{1}$ from $H_{0}$. First, we assume that the adversary asks only one token query. The general case follows from a standard hybrid argument. Note that the two circuits $C[k]$ and $U\left[C, k, m_{0}, m_{1}, s_{0}, s_{1}\right]$ compute the same function. In fact, on input $m=m_{b}$ for $b \in\{0,1\}$ the first circuit computes $C\left(m_{b}|| \mathrm{F}\right.$.Eval $\left.\left(\mathrm{k}, \mathrm{m}_{\mathrm{b}}\right)\right)$ and the second circuit computes $C\left(m_{b} \| s_{b}\right)$ that, by construction of $s_{b}$, equals $C\left(m_{b}|| \mathrm{F}\right.$. Eval $\left.\left(\mathrm{k}, \mathrm{m}_{\mathrm{b}}\right)\right)$. For any other input $m \neq m_{0}, m_{1}$, by construction, the two circuits output the same value as well. Then, consider the two ensembles (parameterized by the security parameter $\lambda$ ) of distributions $D_{0}$ and $D_{1}$ defined so to output with probability 1 , respectively, the circuit $C[k]$ and the circuit $U\left[C, k, m_{0}, m_{1}, s_{0}, s_{1}\right]$. Notice that such pair of ensembles of distributions is feasible, thus the claim follows from the INDFP-Security of FPFE.

- $H_{2}$. This experiment is identical to $H_{1}$ except that any token for randomized circuit $C$ is computed as FPFE.KGen(Msk, $\left.U\left[C, k\left(\left\{m_{0}, m_{1}\right\}\right), m_{0}, m_{1}, s_{0}, s_{1}\right]\right)$ where $s_{b}=$ F.Eval $\left(\mathrm{k}, \mathrm{m}_{\mathrm{b}}\right)$ for $b \in\{0,1\}$ as before but $k\left(\left\{m_{0}, m_{1}\right\}\right)=\mathrm{F}$.Pnct $\left(\mathrm{k},\left\{\mathrm{m}_{0}, \mathrm{~m}_{1}\right\}\right)$ and $U\left[C, k\left(\left\{m_{0}, m_{1}\right\}, m_{0}, m_{1}, s_{0}, s_{1}\right]\right.$ is identical to $U\left[C, k, m_{0}, m_{1}, s_{0}, s_{1}\right]$ except for the constant $k\left(\left\{m_{0}, m_{1}\right\}\right.$ instead of $k$.

Claim 5.4. Indistinguishability of $H_{2}$ from $H_{1}$. First, we assume that the adversary asks only one token query. The general case follows from a standard hybrid argument. Note that the two circuits $U\left[C, k, m_{0}, m_{1}, s_{0}, s_{1}\right]$ and $U\left[C, k\left(\left\{m_{0}, m_{1}\right\}\right), m_{0}, m_{1}, s_{0}, s_{1}\right]$ differ only for the constant values $k$ and $k\left(\left\{m_{0}, m_{1}\right\}\right)$. By the fact that $\mathrm{F}$ preserves the functionality at points different from the punctured points, the two circuits compute the same function. Thus, as argued above, the claim follows from the INDFP-Security of FPFE.

- $H_{3}$. This experiment is identical to $H_{2}$ except that any token for randomized circuit $C$ is computed as FPFE.KGen(Msk, $\left.U\left[C, k\left(\left\{m_{0}, m_{1}\right\}\right), m_{0}, m_{1}, s_{0}, s_{1}\right]\right)$ where $s_{0}$ and $s_{1}$ are randomly and independently chosen in $\{0,1\}^{m(\lambda)}$, and $k=\mathrm{F} . \operatorname{Key}\left(1^{\lambda}\right)$ and $k\left(\left\{m_{0}, m_{1}\right\}\right)=$ F.Pnct $\left(k,\left\{m_{0}, m_{1}\right\}\right)$ are as in the previous experiments. 
Claim 5.5. Indistinguishability of $\mathrm{H}_{3}$ from $\mathrm{H}_{2}$. First, we assume that the adversary asks only one token query. The general case follows from a standard hybrid argument. The indistinguishability of the two experiments follows from the pseudorandomness of $F$ at the punctured points $m_{0}$ and $m_{1}$.

- $H_{4}$. This experiment is identical to $H_{3}$ except that the challenge ciphertext is computed as encryption of $m_{1}$.

Claim 5.6. Indistinguishability of $\mathrm{H}_{4}$ from $\mathrm{H}_{3}$. First, we notice what follows. Any token for randomized circuit $C$ for which $\mathcal{A}$ asked a query is computed as

FPFE.KGen(Msk, $\left.U\left[C, k\left(\left\{m_{0}, m_{1}\right\}\right), m_{0}, m_{1}, s_{0}, s_{1}\right]\right)$ where $s_{0}$ and $s_{1}$ are randomly and independently chosen in $\{0,1\}^{m(\lambda)}$ and $k\left(\left\{m_{0}, m_{1}\right\}\right)=$ F.Pnct $\left(\mathrm{k},\left\{\mathrm{m}_{0}, \mathrm{~m}_{1}\right\}\right)$ (for $k$ computed as $\left.k \leftarrow \mathrm{F} . \mathrm{Key}\left(1^{\lambda}\right)\right)$. So we have $U\left[C, k\left(\left\{m_{0}, m_{1}\right\}\right), m_{0}, m_{1}, s_{0}, s_{1}\right]\left(m_{0}\right) \triangleq C\left(m_{0} ; s_{0}\right)$ and $U\left[C, k\left(\left\{m_{0}, m_{1}\right\}\right), m_{0}, m_{1}, s\right]\left(m_{1}\right) \triangleq C\left(m_{1} ; s_{1}\right)$. Sincd $\mathcal{A}$ is a legitimate RFE adversary, then $\mathcal{A}$ only asks queries for circuits $C$ such that $C\left(m_{0} ; s\right)$ is statistically indistinguishable from $C\left(m_{1} ; s\right)$ where the probability is taken over the choices of $s$ and thus the above equations imply that with all except negligible probability over the choices of $s_{0}$ and $s_{1}$ in $\{0,1\}^{m(\lambda)}, C\left(m_{0} ; s_{0}\right)=C\left(m_{1} ; s_{1}\right)$. Therefore, the indistinguishability of the two experiments follows from the IND-Security of FPFE.

- $H_{5}$. This experiment is identical to $H_{4}$ except that any token for randomized circuit $C$ is computed as FPFE.KGen(Msk, $\left.U\left[C, k\left(\left\{m_{0}, m_{1}\right\}\right), m_{0}, m_{1}, s_{0}, s_{1}\right]\right)$ where $s_{b}$ for $b \in\{0,1\}$ is computed as F.Eval $\left(\mathrm{k}, \mathrm{m}_{\mathrm{b}}\right)$, and $k=\mathrm{F} . \mathrm{Key}\left(1^{\lambda}\right)$ and $k\left(\left\{m_{0}, m_{1}\right\}\right)=\mathrm{F}$.Pnct $\left(\mathrm{k},\left\{\mathrm{m}_{0}, \mathrm{~m}_{1}\right\}\right)$ are as in the previous experiments.

Claim 5.7. Indistinguishability of $H_{5}$ from $H_{4}$. The indistinguishability of the two experiments is symmetrical to that of $\mathrm{H}_{3}$ from $\mathrm{H}_{2}$.

- $H_{6}$. This experiment is identical to $H_{5}$ except that any token for randomized circuit $C$ is computed as FPFE.KGen(Msk, $\left.U\left[C, k, m_{0}, m_{1}, s_{0}, s_{1}\right]\right)$ where $s_{b}$ for $b \in\{0,1\}$ is computed as $\mathrm{F} . \operatorname{Eval}\left(\mathrm{k}, \mathrm{m}_{\mathrm{b}}\right)$ and $k=\mathrm{F} . \operatorname{Key}\left(1^{\lambda}\right)$ as in the previous experiments.

Claim 5.8. Indistinguishability of $H_{6}$ from $H_{5}$. The indistinguishability of the two experiments is symmetrical to that of $\mathrm{H}_{2}$ from $H_{1}$.

- $H_{7}$. This experiment is identical to $H_{6}$ except that any token for randomized circuit $C$ is computed as FPFE.KGen(Msk, $C[k])$ where $k=\mathrm{F} . \mathrm{Key}\left(1^{\lambda}\right)$ as in the previous experiments.

Claim 5.9. Indistinguishability of $H_{7}$ from $H_{6}$. The indistinguishability of the two experiments is symmetrical to that of $H_{1}$ from $H_{0}$.

Note that experiments $H_{0}$ and $H_{7}$ correspond to the experiments of INDRFE-Security where the challenger encrypts respectively $m_{0}$ and $m_{1}$. Thus, the theorem holds. I

\section{Relation between Primitives}

It is easy to see that quasi-siO implies iO that in turn is known to imply FE. Thus, quasi-siO implies FPFE. Moreover, FAS can be used to construct a quasi-siO as follows. An obfuscation of circuit 
$C$ will consist of a signature for $C$ and the verification key of the FAS scheme, and to evaluate the obfuscated circuit on an input $x$, just run the verification algorithm of FAS with input the verification key, the signature and the message $m$. From the anonymity of FAS, such obfuscator is easily seen to be a quasi-siO.

Since FPFE implies FPABE, that in turn implies FAS, we have that FAS, FPFE and quasi-siO are equivalent primitives. The equivalence also extends to FPRFE.

One of the key points highlighted by our results is that FPABE implies quasi-siO and thus $\mathrm{iO}$ that in turn implies FE [26], a notable fact that sheds light on the importance and power of function privacy for FE. In Figure 2, we present relations among the primitives studied or discussed in this paper, except for the implication presented in Section 1.4 about IPE.

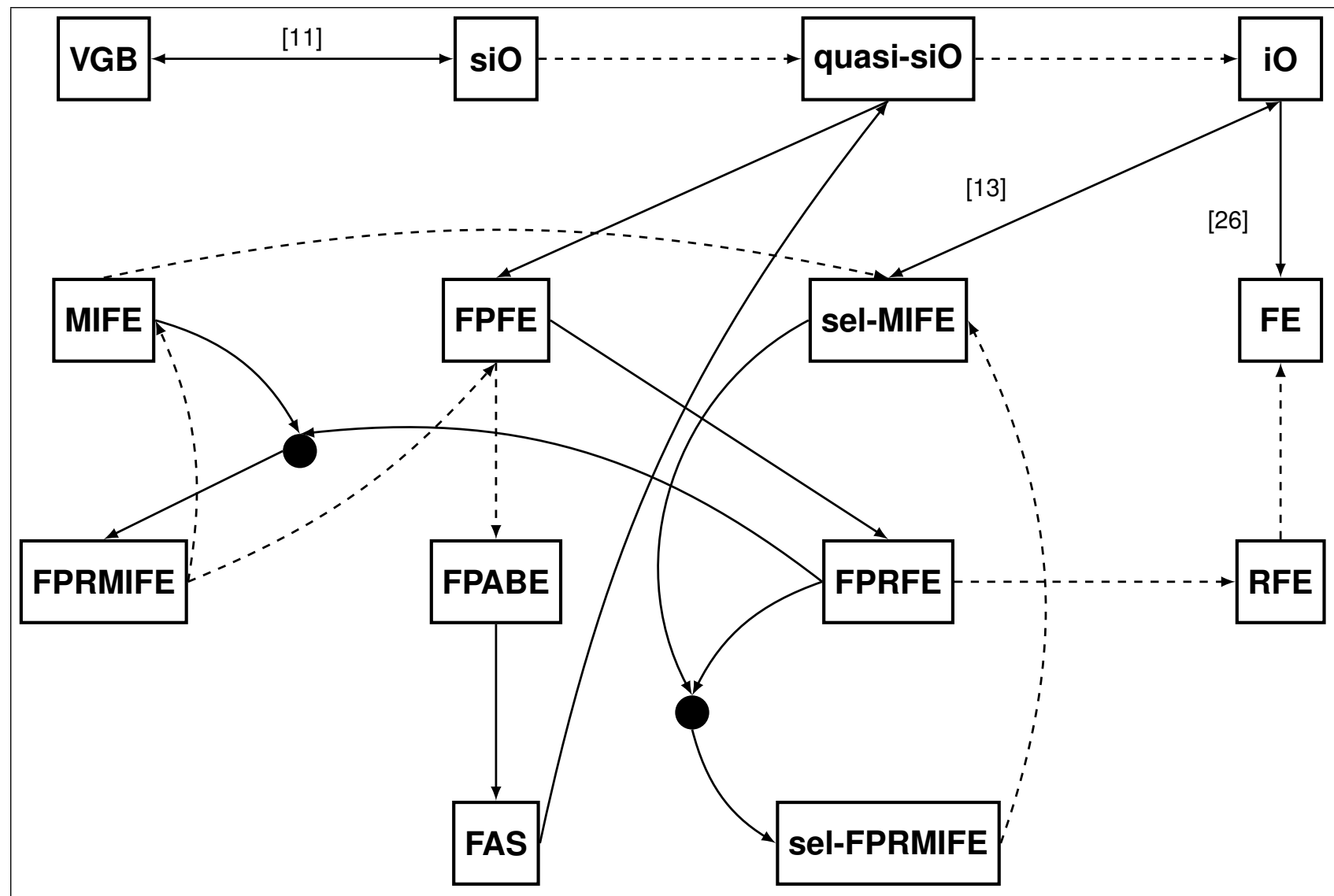

Fig. 1. Relations among primitives studied or discussed in this paper (except for the implication of Section 1.4): A line with arrow from A to $B$ denotes that it is possible to build $B$ from $A$ and lines are annotated with the work where the implication first appeared, or unlabeled if such implication is discussed in this paper. A line from A to $B$ with arrows at both ends denotes that it is possible to build A from $B$ and vice-versa. A dashed line denotes a trivial implication. Two lines coming respectively from $A$ and $B$ with arrow directed in a circled black box with an outgoing line with arrow directed to box $C$ means that it is possible to build $C$ assuming both $A$ and $B$ (e.g., FPRFE and MIFE imply FPRMIFE). For FE and MIFE we assume adaptive indistinguishability-security. For the security of FPFE, FAS and RFE see Section 2. FPRFE denotes a RFE scheme with a standard form of function privacy for deterministic circuits (see Section 5) and FPRMIFE denotes a FPRFE scheme that is in addition multi-inputs. 


\section{Acknowledgments}

Vincenzo Iovino is supported by a CORE (junior track) grant (no. 11299247) of the Luxembourg National Research Fund. Qiang Tang's work was carried out when he worked at University of Luxembourg, and it was supported by a CORE (junior track) grant from the Luxembourg National Research Fund.

\section{References}

[1] Dan Boneh, Amit Sahai, and Brent Waters. Functional encryption: Definitions and challenges. In Yuval Ishai, editor, TCC 2011: 8th Theory of Cryptography Conference, volume 6597 of Lecture Notes in Computer Science, pages 253-273, Providence, RI, USA, March 28-30, 2011. Springer, Berlin, Germany.

[2] Dan Boneh, Giovanni Di Crescenzo, Rafail Ostrovsky, and Giuseppe Persiano. Public key encryption with keyword search. In Christian Cachin and Jan Camenisch, editors, Advances in Cryptology - EUROCRYPT 2004, volume 3027 of Lecture Notes in Computer Science, pages 506-522, Interlaken, Switzerland, May 2-6, 2004. Springer, Berlin, Germany.

[3] Dan Boneh and Brent Waters. Conjunctive, subset, and range queries on encrypted data. In Salil P. Vadhan, editor, TCC 2007: 4th Theory of Cryptography Conference, volume 4392 of Lecture Notes in Computer Science, pages 535-554, Amsterdam, The Netherlands, February 21-24, 2007. Springer, Berlin, Germany.

[4] Jonathan Katz, Amit Sahai, and Brent Waters. Predicate encryption supporting disjunctions, polynomial equations, and inner products. In Nigel P. Smart, editor, Advances in Cryptology - EUROCRYPT 2008, volume 4965 of Lecture Notes in Computer Science, pages 146-162, Istanbul, Turkey, April 13-17, 2008. Springer, Berlin, Germany.

[5] Sanjam Garg, Craig Gentry, Shai Halevi, Mariana Raykova, Amit Sahai, and Brent Waters. Candidate indistinguishability obfuscation and functional encryption for all circuits. In 54th Annual IEEE Symposium on Foundations of Computer Science, FOCS 2013, 26-29 October, 2013, Berkeley, CA, USA, pages 40-49. IEEE Computer Society, 2013.

[6] Emily Shen, Elaine Shi, and Brent Waters. Predicate privacy in encryption systems. In Omer Reingold, editor, TCC 2009: 6th Theory of Cryptography Conference, volume 5444 of Lecture Notes in Computer Science, pages 457-473. Springer, Berlin, Germany, March 15$17,2009$.

[7] Zvika Brakerski and Gil Segev. Function-private functional encryption in the private-key setting. In Theory of Cryptography - 12th Theory of Cryptography Conference, TCC 2015, Warsaw, Poland, March 23-25, 2015, Proceedings, Part II, pages 306-324, 2015.

[8] Dan Boneh, Ananth Raghunathan, and Gil Segev. Function-private identity-based encryption: Hiding the function in functional encryption. In Ran Canetti and Juan A. Garay, editors, Advances in Cryptology - CRYPTO 2013 - 33rd Annual Cryptology Conference, Santa Barbara, CA, USA, August 18-22, 2013. Proceedings, Part II, volume 8043 of Lecture Notes in Computer Science, pages 461-478. Springer, 2013. 
[9] Dan Boneh, Ananth Raghunathan, and Gil Segev. Function-private subspace-membership encryption and its applications. In Advances in Cryptology - ASIACRYPT 2013 - 19th International Conference on the Theory and Application of Cryptology and Information Security, Bengaluru, India, December 1-5, 2013, Proceedings, Part I, pages 255-275, 2013.

[10] Shashank Agrawal, Shweta Agrawal, Saikrishna Badrinarayanan, Abishek Kumarasubramanian, Manoj Prabhakaran, and Amit Sahai. On the practical security of inner product functional encryption. In Public-Key Cryptography - PKC 2015 - 18th IACR International Conference on Practice and Theory in Public-Key Cryptography, Gaithersburg, MD, USA, March 30 - April 1, 2015, Proceedings, pages 777-798, 2015.

[11] Nir Bitansky, Ran Canetti, Yael Tauman Kalai, and Omer Paneth. On virtual grey box obfuscation for general circuits. In Advances in Cryptology - CRYPTO 2014 - 34th Annual Cryptology Conference, Santa Barbara, CA, USA, August 17-21, 2014, Proceedings, Part II, pages 108-125, 2014.

[12] Mihir Bellare, Igors Stepanovs, and Stefano Tessaro. Contention in cryptoland: Obfuscation, leakage and UCE. In Theory of Cryptography - 13th International Conference, TCC 2016-A, Tel Aviv, Israel, January 10-13, 2016, Proceedings, Part II, pages 542-564, 2016.

[13] Shafi Goldwasser, S. Dov Gordon, Vipul Goyal, Abhishek Jain, Jonathan Katz, Feng-Hao Liu, Amit Sahai, Elaine Shi, and Hong-Sheng Zhou. Multi-input functional encryption. In Phong Q. Nguyen and Elisabeth Oswald, editors, Advances in Cryptology - EUROCRYPT 2014 - 33rd Annual International Conference on the Theory and Applications of Cryptographic Techniques, Copenhagen, Denmark, May 11-15, 2014. Proceedings, volume 8441 of Lecture Notes in Computer Science, pages 578-602. Springer, 2014.

[14] Dan Boneh and Matthew K. Franklin. Identity-based encryption from the Weil pairing. In Joe Kilian, editor, Advances in Cryptology - CRYPTO 2001, volume 2139 of Lecture Notes in Computer Science, pages 213-229, Santa Barbara, CA, USA, August 19-23, 2001. Springer, Berlin, Germany.

[15] Vipul Goyal, Omkant Pandey, Amit Sahai, and Brent Waters. Attribute-based encryption for fine-grained access control of encrypted data. In Ari Juels, Rebecca N. Wright, and Sabrina De Capitani di Vimercati, editors, ACM CCS 06: 13th Conference on Computer and Communications Security, pages 89-98, Alexandria, Virginia, USA, October 30 - November 3, 2006. ACM Press. Available as Cryptology ePrint Archive Report 2006/309.

[16] Elette Boyle, Shafi Goldwasser, and Ioana Ivan. Functional signatures and pseudorandom functions. In Public-Key Cryptography - PKC 2014 - 17th International Conference on Practice and Theory in Public-Key Cryptography, Buenos Aires, Argentina, March 26-28, 2014. Proceedings, pages 501-519, 2014.

[17] Ran Canetti. Towards realizing random oracles: Hash functions that hide all partial information. In Burton S. Kaliski Jr., editor, Advances in Cryptology - CRYPTO'97, volume 1294 of Lecture Notes in Computer Science, pages 455-469, Santa Barbara, CA, USA, August 17-21, 1997. Springer, Berlin, Germany.

[18] Alexander W. Dent, Marc Fischlin, Mark Manulis, Martijn Stam, and Dominique Schröder. Confidential signatures and deterministic signcryption. In Phong Q. Nguyen and David 
Pointcheval, editors, PKC 2010: 13th International Conference on Theory and Practice of Public Key Cryptography, volume 6056 of Lecture Notes in Computer Science, pages 462479, Paris, France, May 26-28, 2010. Springer, Berlin, Germany.

[19] Vipul Goyal, Abhishek Jain, Venkata Koppula, and Amit Sahai. Functional encryption for randomized functionalities. In Theory of Cryptography - 12th Theory of Cryptography Conference, TCC 2015, Warsaw, Poland, March 23-25, 2015, Proceedings, Part II, pages 325$351,2015$.

[20] Tatsuaki Okamoto and Katsuyuki Takashima. Adaptively attribute-hiding (hierarchical) inner product encryption. In David Pointcheval and Thomas Johansson, editors, Advances in Cryptology - EUROCRYPT 2012, volume 7237 of Lecture Notes in Computer Science, pages 591-608, Cambridge, UK, April 15-19, 2012. Springer, Berlin, Germany.

[21] Shweta Agrawal, David Mandell Freeman, and Vinod Vaikuntanathan. Functional encryption for inner product predicates from learning with errors. In Dong Hoon Lee and Xiaoyun Wang, editors, Advances in Cryptology - ASIACRYPT 2011, volume 7073 of Lecture Notes in Computer Science, pages 21-40, Seoul, South Korea, December 4-8, 2011. Springer, Berlin, Germany.

[22] Shashank Agrawal, Shweta Agrawal, Saikrishna Badrinarayanan, Abishek Kumarasubramanian, Manoj Prabhakaran, and Amit Sahai. Function private functional encryption and property preserving encryption : New definitions and positive results. Cryptology ePrint Archive, 2013. http://eprint.iacr.org/2013/744.

[23] Amit Sahai and Brent Waters. How to use indistinguishability obfuscation: Deniable encryption, and more. In Symposium on Theory of Computing Conference, STOC'14, New York, NY, USA, 31 May-3 June, 2014, pages 475-484, 2014.

[24] Nir Bitansky, Ran Canetti, Yael Tauman Kalai, and Omer Paneth. On virtual grey box obfuscation for general circuits. IACR Cryptology ePrint Archive, 2014. http://eprint. iacr. org/2014/554/20140805:181558. Note that we refer to the version posted on 14 August 2014.

[25] Ilan Komargodski, Gil Segev, and Eylon Yogev. Functional encryption for randomized functionalities in the private-key setting from minimal assumptions. In Theory of Cryptography 12th Theory of Cryptography Conference, TCC 2015, Warsaw, Poland, March 23-25, 2015, Proceedings, Part II, pages 352-377, 2015.

[26] Brent Waters. A punctured programming approach to adaptively secure functional encryption. In Advances in Cryptology - CRYPTO 2015 - 35th Annual Cryptology Conference, Santa Barbara, CA, USA, August 16-20, 2015, Proceedings, Part II, pages 678-697, 2015. 


\section{A. Security of FE}

The indistinguishability-based notion of security for functional encryption scheme $\mathrm{FE}=$ (Setup, KGen, Enc, Eval) for functionality $F$ defined over $(K, M)$ is formalized by means of the following game IND $\mathcal{F}_{\mathcal{A}}^{\mathrm{FE}}$ between an adversary $\mathcal{A}=\left(\mathcal{A}_{0}, \mathcal{A}_{1}\right)$ and a challenger $\mathcal{C}$. Below, we present the definition for only one message; it is easy to see the definition extends naturally for multiple messages.

$\operatorname{IND}_{\mathcal{A}}^{\mathrm{FE}}\left(1^{\lambda}\right)$

1. $\mathcal{C}$ generates $(\mathrm{Pk}, \mathrm{Msk}) \leftarrow \operatorname{Setup}\left(1^{\lambda}\right)$ and runs $\mathcal{A}_{0}$ on input $\mathrm{Pk}$;

2. $\mathcal{A}_{0}$ submits queries for keys $k_{i} \in K$ for $i=1, \ldots, q_{1}$ and, for each such query, $\mathcal{C}$ computes $\operatorname{Tok}_{i}=\mathrm{KGen}\left(\mathrm{Msk}, k_{i}\right)$ and sends it to $\mathcal{A}_{0}$.

When $\mathcal{A}_{0}$ stops, it outputs two challenge plaintexts $m_{0}, m_{1} \in M$ satisfying $\left|m_{0}\right|=$ $\left|m_{1}\right|$ and its internal state st.

3. $\mathcal{C}$ picks $b \in\{0,1\}$ at random, computes the challenge ciphertext $\mathrm{Ct}=\mathrm{Enc}\left(\mathrm{Pk}, m_{b}\right)$ and sends $\mathrm{Ct}$ to $\mathcal{A}_{1}$ that resumes its computation from state st.

4. $\mathcal{A}_{1}$ submits queries for keys $k_{i} \in K$ for $i=q_{1}+1, \ldots, q$ and, for each such query, $\mathcal{C}$ computes $\operatorname{Tok}_{i}=\mathrm{KGen}\left(\mathrm{Msk}, k_{i}\right)$ and sends it to $\mathcal{A}_{1}$.

5. When $\mathcal{A}_{1}$ stops, it outputs $b^{\prime}$.

6. Output: if $b=b^{\prime}, m_{0}$ and $m_{1}$ are of the same length, and $F\left(k_{i}, m_{0}\right)=F\left(k_{i}, m_{1}\right)$ for $i=1 \ldots, q$, then output 1 else output 0 .

The advantage of adversary $\mathcal{A}$ in the above game is defined as

$$
\operatorname{Adv}_{\mathcal{A}}^{\mathrm{FE}, \mathrm{IND}}\left(1^{\lambda}\right)=\left|\operatorname{Prob}\left[\operatorname{IND}_{\mathcal{A}}^{\mathrm{FE}}\left(1^{\lambda}\right)=1\right]-1 / 2\right| .
$$

Definition A.1. We say that FE is indistinguishably secure (IND-Ssecure, for short) if all nonuniform families of PPT adversaries $\mathcal{A}=\left\{\mathcal{A}_{\lambda}\right\}_{\lambda \in \mathbb{N}}$ have at most negligible advantage in the above game. 


\section{A. Figure}

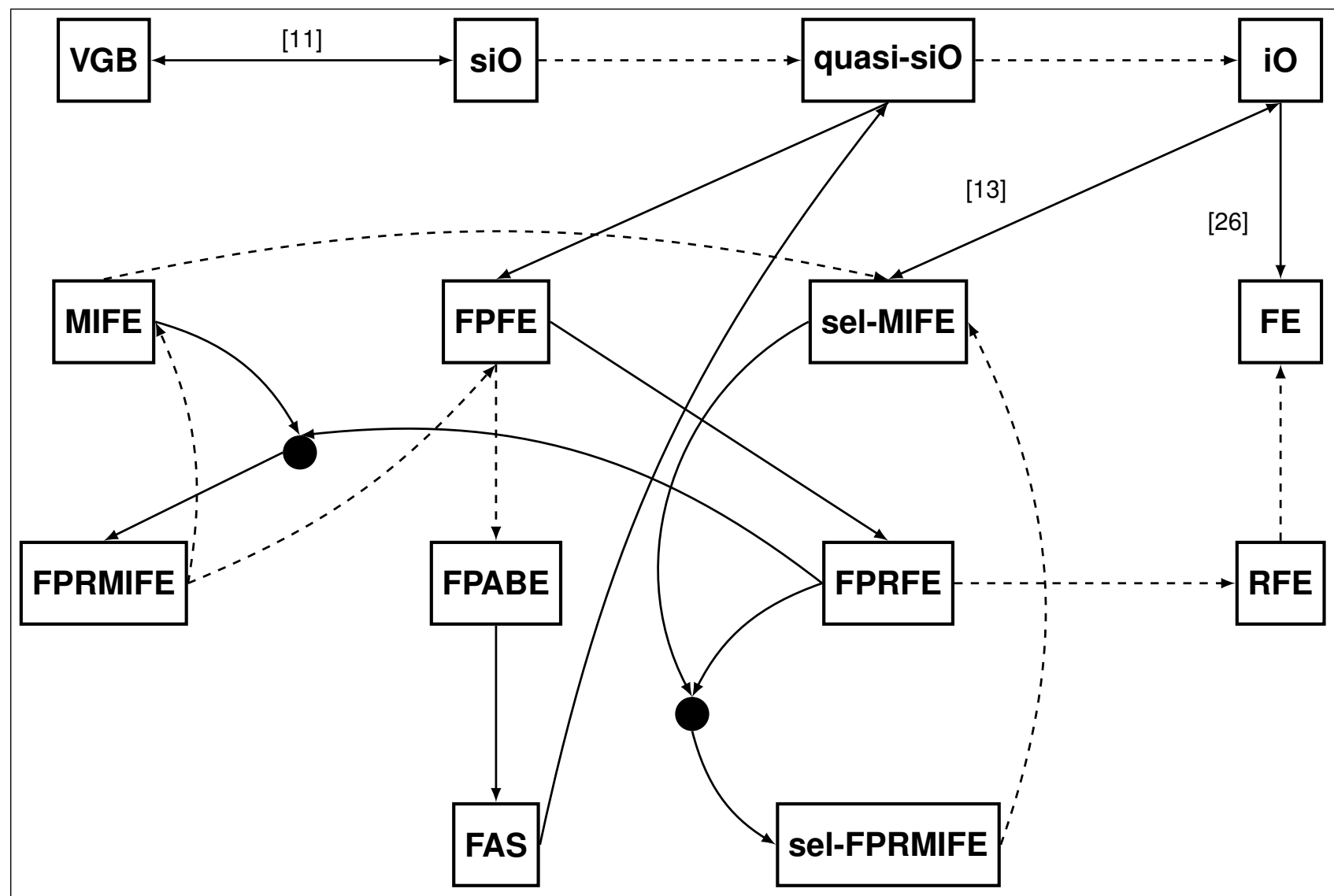

Fig. 2. Relations among primitives studied or discussed in this paper (except for the implication of Section 1.4): A line with arrow from $A$ to $B$ denotes that it is possible to build $B$ from $A$ and lines are annotated with the work where the implication first appeared, or unlabeled if such implication is discussed in this paper. A line from A to $B$ with arrows at both ends denotes that it is possible to build $A$ from $B$ and vice-versa. A dashed line denotes a trivial implication. Two lines coming respectively from $A$ and $B$ with arrow directed in a circled black box with an outgoing line with arrow directed to box $C$ means that it is possible to build $C$ assuming both A and B (e.g., FPRFE and MIFE imply FPRMIFE). For FE and MIFE we assume adaptive indistinguishability-security. For the security of FPFE, FAS and RFE see Section 2. FPRFE denotes a RFE scheme with a standard form of function privacy for deterministic circuits (see Section 5) and FPRMIFE denotes a FPRFE scheme that is in addition multi-inputs. 


\section{B. Proofs and security reductions}

In this section we include the proofs that we did not include in the main body.

\section{B.1. Adaptively-secure function-private IPE $\rightarrow$ adaptively-secure DNFEnc}

Now we prove that an adaptively-secure function-private IPE implies an adaptively-secure DNFEnc (see Section 1.4).

Proof: For simplicity we will consider only a single conjunction and one token query. At the end we show how to remove such restrictions. Hereafter, we also assume that the reader be familiar with the Katz et al.'s transformation presented in Section 1.4.

Observe that the reduction from IPE to DNFEnc fails only in the case that the adversary for the IND-Security against the DNFEnc scheme is able to output as challenge a pair of values $\left(x_{1}, x_{2}\right)$ such that $\operatorname{AND}_{I_{1}, I_{2}}\left(x_{1}, x_{2}\right)=0$ but $p\left(x_{1}, x_{2}\right)=0$. Thus, we have to show that for any PPT adversary the probability that this event occur is negligible in the security parameter. Towards a contradiction we suppose that there exist an adversary $\mathcal{A}$ that during the IND-Security experiment against the DNFEnc scheme is able to output a challenge with such values $x_{1}$ and $x_{2}$ with some non-negligible probability $\alpha(n)$ (for simplicity hereafter we do not explicitly mention the payload messages).

Assume that for any value of the security parameter $n$ our DNFEnc scheme is parameterized by a prime $p_{n}$ of length $n$ that induces the field $\mathbb{Z}_{p_{n}}$ over which the variables are defined. Given a conjunction $\phi \triangleq \operatorname{AND}_{I_{1}, I_{2}}$ we call $p_{\phi, r}$ the corresponding polynomial that uses randomness $r$ as specified in Section 1.4 (recall that here for simplicity we restrict the analysis to formulae consisting of a single conjunction) and we call $C_{\phi, r}$ the circuit that evaluates the predicate $C_{\phi, r}\left(x_{1}, x_{2}\right)=1$ if and only if $p_{\phi, r}\left(x_{1}, x_{2}\right)=0$.

Given a conjunction $\phi$ consider the pair of ensembles of distributions $D_{0}$ and $D_{1}$ such that for each value of the security parameter $n, D_{0, n}$ outputs $C_{\phi, r}^{0}$ for a randomly chosen element $r$ in $\mathbb{Z}_{p_{n}}$ and $D_{1, n}$ outputs $C_{\phi, r}^{1}$ for an element $r$ chosen such that its first $n$ bits are set to 0 and the remaining are randomly selected.

It is easy to see that $D_{0}$ and $D_{1}$ are a pair of feasible entropy distributions (and in addition they are efficiently samplable). In fact, on any input $\left(x_{1}, x_{2}\right)$ such that $\operatorname{AND}_{I_{1}, I_{2}}\left(x_{1}, x_{2}\right)=1$ the two circuits output by the two distributions give the same answer (i.e., 1) and the adversary can find an input $\left(x_{1}, x_{2}\right)$ such that $\operatorname{AND}_{I_{1}, I_{2}}\left(x_{1}, x_{2}\right)=0$ but $C^{0}\left(x_{1}, x_{2}\right)=1$ with probability at most $2^{-n} \cdot q$, where $q(\cdot)$ is the number of oracle queries, and such that $\operatorname{AND}_{I_{1}, I_{2}}\left(x_{1}, x_{2}\right)=0$ but $C^{1}\left(x_{1}, x_{2}\right)=1$ with probability at most $2^{-n / 2} \cdot q$; thus any adversary with a polynomial number of queries can have only negligible advantage in distinguishing oracle access to the two distribution ensembles.

Now observe that the INDFP-Security guarantees that no PPT adversary can tell apart a token for the circuit $C^{0}$ from a token for the circuit $C^{1}$. So, we construct an adversary $\mathcal{B}$ against the INDFPSecurity of DNFEnc that makes use of the adversary $\mathcal{A}$ against its IND-Security. $\mathcal{B}$ simulates the environment to $\mathcal{B}$ receiving from its challenger either a token for $C_{\phi, r}^{0}$ chosen by $D_{0}$ or a token for $C_{\phi, r}^{1}$ chosen by $D_{1}$. When $\mathcal{A}$ outputs its challenge $\left(x_{1}, x_{2}\right), \mathcal{B}$ checks 1$)$ that the token evaluates on a ciphertext for this challenge to 1 (thus implying, by the correctness of the polynomial evaluation scheme, that $\left.\left.p_{\phi, r}\left(x_{1}, x_{2}\right)=0\right), 2\right) \operatorname{AND}_{I_{1}, I_{2}}\left(x_{1}, x_{2}\right)=0$ and if both checks are verified $\mathcal{B}$ computes $r^{\prime} \triangleq-\left(x_{2}-I_{2}\right) /\left(x_{1}-I_{1}\right)$ (notice that if condition 1) and 2) are satisfied then it cannot be $x_{1}=I_{1}$ ) and finally output 0 (indicating a guess for the circuit $C^{0}$ ) if and only if the first $n / 2$ bits of $p$ are 
different from 0 ; if one of the previous checks is not satisfies $\mathcal{B}$ outputs 1 (indicating a guess for the circuit $C^{1}$ ).

If the circuit was chosen from $D_{0}$ then $\mathcal{B}$ outputs 0 with probability at least $\alpha(n)$, up to a negligible factor, whereas if the circuit was chosen from $D_{1}$ then $\mathcal{B}$ outputs 0 with probability 0 . Therefore, $\mathcal{B}$ can win in the INDFP-Security game against DNFEnc, a contradiction.

For the general case of DNF formulae (instead of formulae consisting of a single conjunction) observe that a DNF formula, when implemented with the Katz et al.'s transformation consists in a product of polynomial $p_{\phi, r}$ 's (see Section 1.4) in which each term uses different randomness (also recall that there is no randomness introduced for the disjunctions). Thus the general case for DNF formulae follows by a standard hybrid argument. The general case for multiple token queries can be handled by a standard hybrid argument as well.

\section{B.2. Proof of Theorem 3.3}

Proof: Suppose that there exists a legitimate function privacy adversaries $\mathcal{A}=\left\{\mathcal{A}_{n}\right\}_{n \in \mathbb{N}}$ breaking the INDFP-Security of FPFE[qsiO, FE]. Specifically, suppose that there exists a non-negligible function $p(\cdot)$ such that for any $n \in \mathbb{N}, \mathcal{A}_{n}$ wins in the INDFP-Security parameterized by $n$ with advantage $\geq p(n)$.

Thus, by an averaging argument, for any $n \in \mathbb{N}$ there exist two distributions $D_{0, n}$ and $D_{1, n}$ and random strings $r_{1}, r_{2} \in\{0,1\}^{\star}$ (to be defined later) such that in the the security experiment (for parameter $n$ ) executed with random strings $r_{1}, r_{2}, \mathcal{A}_{n}$ outputs such distributions as challenge distributions with non-zero probability and under the occurrence of such event $\mathcal{A}_{n}$ has advantage $p(n)$. Precisely, $r_{1}$ is used to compute the public-key and the master secret-key with which the token queries can be answered (w.l.o.g., we can assume that KGen is deterministic) and $r_{2}$ is used to run the adversary until the challenge query (that is, after the challenge query other randomness will be used and $r_{1}$ and $r_{2}$ determine the behavior of $\mathcal{A}_{n}$ until that point but not after. ${ }^{7}$ ).

Then, from the fact that $\mathcal{A}$ is a legitimate function privacy adversary it follows that the ensembles $D_{0}=\left\{D_{0, n}\right\}_{n \in \mathbb{N}}$ and $D_{1}=\left\{D_{1, n}\right\}_{n \in \mathbb{N}}$ are a pair of ensembles of feasible entropy distributions and thus it is straightforward to construct a family of non-uniform distinguishers $\mathcal{D}=\left\{\mathcal{D}_{n}\right\}$ breaking the security of qsiO as follows. Specifically, $\mathcal{D}_{n}$ has embedded the random strings $r_{1}, r_{2}$ (that have size polynomial in $n$ ) and takes as input the obfuscated circuit $C^{\prime}$ that is a computed as qsiO $(C)$ where the circuit $C$ is drawn from either $D_{0, n}$ or $D_{1, n} . \mathcal{D}_{n}$ runs the setup of FE with security parameter $n$ and randomness $r_{1}$ to get the public-key Mpk and master secret-key Msk of FE.

Then, $\mathcal{D}_{n}$ runs $\mathcal{A}_{n}$ with randomness $r_{2}$ on input Mpk and answers the $\mathcal{A}_{n}$ 's queries using Msk. Then, by construction of $r_{1}$ and $r_{2}, \mathcal{A}_{n}$ outputs as challenge distributions $D_{0, n}$ and $D_{1, n}$. $\mathcal{D}_{n}$ answers the challenge query returning to $\mathcal{A}_{n}$ the token FE.KGen(Msk, $\left.C^{\prime}\right)$ and then continues the execution of $\mathcal{A}_{n}$ as before. At the end $\mathcal{D}_{n}$ outputs what $\mathcal{A}_{n}$ outputs.

It is easy to see that the advantage of $\mathcal{D}_{n}$ in distinguishing whether the input was an obfuscation of a circuit drawn from $D_{0, n}$ or $D_{1, n}$ is $p(n)$ (note here that the probability is also taken over the choices of the randomness used to compute $C^{\prime}$ that is not known to $\mathcal{D}_{n}$ ). Then, we conclude that

\footnotetext{
${ }^{7}$ Recall that there are two ways to define probabilistic algorithms. One is to feed them with a random string, and one is to give them access to an oracle that returns random bits. Here we can adopt the latter convention and in this case we mean that the oracle uses the bits of $r_{2}$ to answer the queries until the challenge phase, and after that the oracle returns uniformly and independently chosen bits. Furthermore, note that $r_{2}$ is not used to answer the challenge query: indeed, as it will be specified later, the randomness used to answer it is chosen by the challenger of quasi-siO and thus it will be not known to the distinguisher
} 
$\mathcal{D}$ along with the ensembles of distributions $D_{0}=\left\{D_{0, n}\right\}_{n \in \mathbb{N}}$ and $D_{1}=\left\{D_{1, n}\right\}_{n \in \mathbb{N}}$ contradicts the security of qsiO. I

\section{B.3. Proof of Theorem 4.3}

PROOF SKETCH. The proof is almost identical to that of theorem 3.3, thus we omit full details. Suppose that there exists a family of non-uniform PPT adversaries $\mathcal{A}=\left\{\mathcal{A}_{n}\right\}_{n \in \mathbb{N}}$ breaking the anonymity of FAS[FPABE]. Then, it is easy to construct a family of non-uniform PPT adversaries $\mathcal{B}=\left\{\mathcal{B}_{n}\right\}_{n \in \mathbb{N}}$ breaking the security of FPABE. Being $\mathcal{A}$ a legitimate FAS adversary, we can construct $\mathcal{B}_{n}$ identical to the distinguisher $\mathcal{D}_{n}$ in the proof of theorem 3.3 except in the way that $\mathcal{B}_{n}$ has to simulates the view to $\mathcal{A}$ and construct the challenge. This is also straightforward. Then, we conclude that $\mathcal{B}$ contradicts the security of qsiO. 